\title{
Approximate Analytic Solutions for the Two-Phase Stefan Problem Using the Adomian Decomposition Method
}

\author{
Xiao-Ying Qin, ${ }^{1}$ Yue-Xing Duan, ${ }^{2}$ and Mao-Ren Yin $^{3}$ \\ ${ }^{1}$ College of Mathematics, Taiyuan University of Technology, Taiyuan, Shanxi 030024, China \\ ${ }^{2}$ College of Computer Science and Technology, Taiyuan University of Technology, Taiyuan, Shanxi 030024, China \\ ${ }^{3}$ Department of Mathematics, Xin Zhou Teachers University, Xinzhou, Shanxi 034000, China
}

Correspondence should be addressed to Xiao-Ying Qin; qxy62723@163.com

Received 22 January 2014; Accepted 3 June 2014; Published 18 June 2014

Academic Editor: Abdel-Maksoud A. Soliman

Copyright (C) 2014 Xiao-Ying Qin et al. This is an open access article distributed under the Creative Commons Attribution License, which permits unrestricted use, distribution, and reproduction in any medium, provided the original work is properly cited.

\begin{abstract}
An Adomian decomposition method (ADM) is applied to solve a two-phase Stefan problem that describes the pure metal solidification process. In contrast to traditional analytical methods, ADM avoids complex mathematical derivations and does not require coordinate transformation for elimination of the unknown moving boundary. Based on polynomial approximations for some known and unknown boundary functions, approximate analytic solutions for the model with undetermined coefficients are obtained using ADM. Substitution of these expressions into other equations and boundary conditions of the model generates some function identities with the undetermined coefficients. By determining these coefficients, approximate analytic solutions for the model are obtained. A concrete example of the solution shows that this method can easily be implemented in MATLAB and has a fast convergence rate. This is an efficient method for finding approximate analytic solutions for the Stefan and the inverse Stefan problems.
\end{abstract}

\section{Introduction}

Problems in which the solution of a partial differential equation (PDE) or a system of such equations has to satisfy certain conditions on the boundary of a prescribed domain are referred to as boundary value problems. However, in many important cases, the boundary of the domain is not known in advance. As the spatial location of the unknown boundary is determined as a function of time, we call these moving-boundary problems, special case of which is the Stefan problem $[1,2]$. Many problems in physics and engineering can be modeled by the Stefan problems, such as melting of ice and alloy solidification [1], fluid-solid uncatalyzed reactions in chemical engineering [3], and lithium intercalation in an iron phosphate particle during discharge of lithium iron phosphate cells [4].

A variety of analytical and numerical methods have been used to solve moving-boundary problems, including Green's function method [5], the perturbation analysis method [6], the level set method [7], the variational iteration method [8], the finite difference method [9], and the moving mesh, finite element method $[10,11]$. However, these analytical methods are often complicated and very few analytic solutions are available in closed form. Numerical methods cannot provide an analytical expression of the solution and the precision is often not high. Identification of approximate analytic solutions with higher precision for moving-boundary problems may be a good option.

Adomian decomposition method (ADM), developed by Adomian [12], has been widely applied to solve various types of equations involving algebraic, differential, partial differential, integral, and integro-differential operations [1223]. ADM is an efficient method for solving PDEs and systems thereof with various types of boundary conditions. This method involves mathematical derivation and numerical operations. Using ADM, we can decompose the task of solving a PDE into a series of subtasks that can easily be carried out using computation software such as MATLAB. Thus, the overall solution of the PDE can be obtained. 


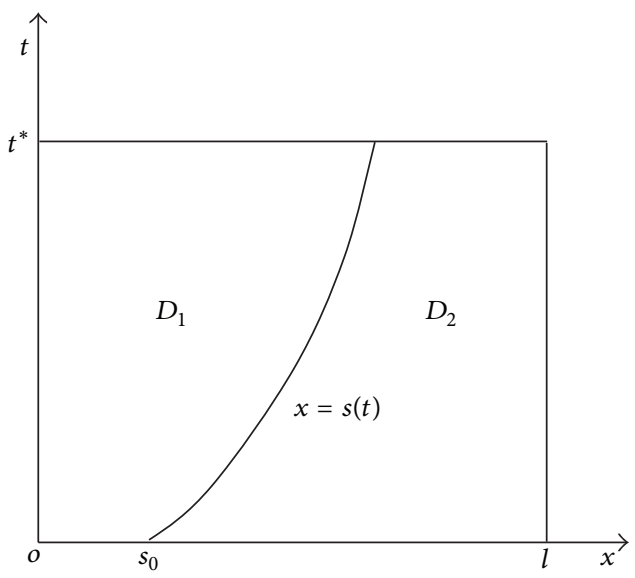

FIGURE 1: The domains of $u=u(x, t)$ and $v=v(x, t)$ and the position of the moving boundary $x=s(t)$ in the domains.

\section{The Two-Phase Stefan Problem}

Solidification of a pure metal can be modeled as a twophase Stefan problem $[1,2,18,24]$, which is a system of ordinary PDEs with an unknown moving boundary. The temperature distribution in the metal liquid phase, $u(x, t)$, and the solid phase, $v(x, t)$, and the moving interface at which solidification occurs, $x=s(t)$, are unknown functions for the model. Functions $u(x, t)$ and $v(x, t)$ satisfy the following heat conduction equations (Figure 1 ):

$$
\begin{aligned}
& \frac{\partial u}{\partial t}=\mu_{1} \frac{\partial^{2} u}{\partial x^{2}}, \quad(x, t) \in D_{1}, \\
& \frac{\partial v}{\partial t}=\mu_{2} \frac{\partial^{2} v}{\partial x^{2}}, \quad(x, t) \in D_{2},
\end{aligned}
$$

where $\mu_{1}$ and $\mu_{2}$ are thermal diffusivity in liquid and solid phases, respectively, and $D_{1}=\{(x, t) \mid 0<x<s(t), 0<t<$ $\left.t^{*}\right\}$ and $D_{2}=\left\{(x, t) \mid s(t)<x<l, 0<t<t^{*}\right\}$ correspond to the liquid- and solid-phase domains $u(x, t)$ and $v(x, t)$, respectively, subject to the initial and boundary conditions

$$
\begin{gathered}
u(x, 0)=\varphi(x), \quad 0 \leq x \leq s_{0}, \\
v(x, 0)=\psi(x), \quad s_{0} \leq x \leq l, \\
-\lambda_{1} \frac{\partial u}{\partial x}(0, t)=q(t), \quad 0 \leq t \leq t^{*}, \\
-\lambda_{2} \frac{\partial v}{\partial x}(l, t)=\alpha(t)\left(v(l, t)-v^{*}\right), \quad 0 \leq t \leq t^{*},
\end{gathered}
$$

where $s_{0}$ is the initial $x$-coordinate of the moving boundary, $\alpha(t)$ is the coefficient of convective heat transfer, $v^{*}$ is the ambient temperature, and $\lambda_{1}$ and $\lambda_{2}$ are thermal conductivity. The moving boundary $s(t)$ is determined by

$$
\begin{gathered}
s(0)=s_{0}, \\
u(s(t), t)=v(s(t), t)=u^{*}, \quad 0 \leq t \leq t^{*}, \\
\kappa \frac{d s}{d t}=\lambda_{2} \frac{\partial v}{\partial x}(s(t), t)-\lambda_{1} \frac{\partial u}{\partial x}(s(t), t), \quad 0<t<t^{*} .
\end{gathered}
$$

The two-phase Stefan problem is modeled by (1)-(7). To use (7) conveniently, we rewrite them as

$$
\begin{gathered}
u\left(s_{0}, 0\right)=v\left(s_{0}, 0\right)=u^{*}, \\
u(s(t), t)=v(s(t), t)=u^{*}, \quad 0<t \leq t^{*}, \\
\lambda_{2} \frac{\partial u}{\partial x}(s(t), t) \frac{\partial v}{\partial x}(s(t), t)-\lambda_{1}\left(\frac{\partial u}{\partial x}\right)^{2}(s(t), t) \\
+\kappa \frac{\partial u}{\partial t}(s(t), t)=0, \quad 0<t<t^{*} .
\end{gathered}
$$

\section{Approximate Analytic Solutions by ADM}

To solve the Stefan problem, coordinate transformation is often used to eliminate the unknown boundary. Grzymkowski and colleagues used the Landau transformation $y=x / s(t)$ to immobilize the boundaries of model (1)-(7) [18]. However, after transformation, the equations and initial boundary conditions for the model become very complicated and may lead to new difficulties in solving the model. In the present study, we avoid using coordinate transformation to solve the model and the task is instead divided into four steps. First, we substitute the Taylor polynomial of $-q(t) / \lambda_{1}$ for $(\partial u / \partial x)(0, t)$ in (5) and substitute polynomials with undetermined coefficients for the unknown $u(0, t), v(l, t)$, and $(\partial v / \partial x)(l, t)$. Second, we find expressions for approximate analytic solutions of (1) and (2) with the unknown parameters using ADM. Third, we substitute the approximate expressions into (6) and (8) to generate a nonlinear algebraic equation system. Fourth, we solve this system of equations to determine the values of the unknown parameters and the approximate analytic solutions of the model.

In operator form, (1) and (2) can be written as

$$
\begin{aligned}
& L_{t} u=\mu_{1} L_{x x} u, \\
& L_{t} v=\mu_{2} L_{x x} v,
\end{aligned}
$$

where $L_{t}$ and $L_{x x}$ are linear operators defined as $L_{t}=\partial / \partial t$ and $L_{x x}=\partial^{2} / \partial x^{2}$. The variation of the two phase temperatures $u(x, t)$ and $v(x, t)$ depends largely on heat transfer at the boundaries $\left\{(x, t) \mid x=0,0 \leq t \leq t^{*}\right\}$ and $\{(x, t) \mid$ $\left.x=l, 0 \leq t \leq t^{*}\right\}$. Therefore, we solve $L_{x x} u$ and $L_{x x} v$ using boundary conditions (5) and (6) and regard the initial conditions (3) and (4) as reference conditions [21]. To obtain solutions satisfying (1), (2), (5), and (6), the $x$-direction is chosen as the search direction and the inverse operators $L_{x x}$ in (9) and (10) are defined as follows:

$$
\begin{aligned}
& L_{x x}^{-1}(\cdot)=\int_{0}^{x}\left[\int_{0}^{w}(\cdot) d y\right] d w, \\
& \bar{L}_{x x}^{-1}(\cdot)=\int_{l}^{x}\left[\int_{l}^{w}(\cdot) d y\right] d w .
\end{aligned}
$$


Applying the inverse operators $L_{x x}^{-1}$ and $\bar{L}_{x x}^{-1}$ to both sides of (9) and (10), respectively, yields

$$
\begin{array}{r}
u(x, t)=\frac{1}{\mu_{1}} L_{x x}^{-1} L_{t} u(x, t)+A(t) x+B(t), \\
v(x, t)=\frac{1}{\mu_{2}} \bar{L}_{x x}^{-1} L_{t} v(x, t)+C(t)(x-l)+D(t),
\end{array}
$$

where $A(t), B(t), C(t)$, and $D(t)$ are undetermined functions. Taking partial derivatives with respect to $x$ on both sides of (12) and using the boundary condition (5) yield

$$
A(t)=-\frac{q(t)}{\lambda_{1}}, \quad 0 \leq t \leq t^{*} .
$$

Letting $x=0$ on both sides of (12) yields

$$
B(t)=u(0, t), \quad 0 \leq t \leq t^{*} .
$$

Similarly, we can obtain

$$
\begin{array}{cc}
C(t)=\frac{\partial v}{\partial x}(l, t), & 0 \leq t \leq t^{*}, \\
D(t)=v(l, t), & 0 \leq t \leq t^{*} .
\end{array}
$$

$B(t), C(t)$, and $D(t)$ are unknown functions. To implement the recursive operation in ADM, we assume that $q(t)$, $u(0, t),(\partial v / \partial x)(l, t)$, and $v(l, t)$ are smooth enough on the interval $\left[0, t^{*}\right]$ so that $A(t), B(t), C(t)$, and $D(t)$ can be approximated by polynomials. Substituting the polynomials $\sum_{k=0}^{n} a_{k} t^{k}, \sum_{k=0}^{n} b_{k} t^{k}, \sum_{k=0}^{n} c_{k} t^{k}$, and $\sum_{k=0}^{n} d_{k} t^{k}$ of degree $n$ for $A(t), B(t), C(t)$, and $D(t)$ in turn in (12) and (13) yields

$$
\begin{gathered}
u(x, t)=\frac{1}{\mu_{1}} L_{x x}^{-1} L_{t} u(x, t)+x \sum_{k=0}^{n} a_{k} t^{k}+\sum_{k=0}^{n} b_{k} t^{k}, \\
v(x, t)=\frac{1}{\mu_{2}} \bar{L}_{x x}^{-1} L_{t} v(x, t)+(x-l) \sum_{k=0}^{n} c_{k} t^{k}+\sum_{k=0}^{n} d_{k} t^{k} .
\end{gathered}
$$

Letting $x=0$ and $t=0$ on both sides of (17) yields

$$
b_{0}=u(0,0)=\varphi(0) \text {. }
$$

Letting $x=l$ and $t=0$ on both sides of (18) yields

$$
d_{0}=v(l, 0)=\psi(l) .
$$

Taking partial derivatives with respect to $x$ on both sides of (18) and then letting $x=l$ and $t=0$ yield

$$
c_{0}=\frac{\partial v}{\partial x}(l, 0) \text {. }
$$

Letting $t=0$ on both sides of (6) yields

$$
-\lambda_{2} \frac{\partial v}{\partial x}(l, 0)=\alpha(0)\left(v(l, 0)-v^{*}\right) .
$$

According to (20), (21), and (22) we can obtain

$$
c_{0}=-\frac{\alpha(0)}{\lambda_{2}}\left(\psi(l)-v^{*}\right) .
$$

The other coefficients of the polynomials $b_{k}, c_{k}$, and $d_{k}(k=$ $1,2, \ldots, n)$ are undetermined constants. According to ADM, we can decompose the unknown functions $u=u(x, t)$ and $v=v(x, t)$ into infinite series forms:

$$
\begin{aligned}
& u=\sum_{n=0}^{\infty} u_{n}, \\
& v=\sum_{n=0}^{\infty} v_{n} .
\end{aligned}
$$

Substituting (24) and (25) into (17) and (18), respectively, and choosing the initial items $u_{0}$ and $v_{0}$ yield the following recursive relations:

$$
\begin{gathered}
u_{0}=x \sum_{k=0}^{n} a_{k} t^{k}+\sum_{k=0}^{n} b_{k} t^{k}, \\
v_{0}=(x-l) \sum_{k=0}^{n} c_{k} t^{k}+\sum_{k=0}^{n} d_{k} t^{k}, \\
u_{m}=\frac{1}{\mu_{1}} L_{x x}^{-1} L_{t} u_{m-1}, \\
v_{m}=\frac{1}{\mu_{2}} \bar{L}_{x x}^{-1} L_{t} v_{m-1},
\end{gathered}
$$

where $m \geq 1$. This leads to the following successive components:

$$
\begin{aligned}
& u_{m}= \frac{x^{2 m}}{\mu_{1}^{m}(2 m) !} \\
& \times\left[\frac{x}{2 m+1} \sum_{k=m}^{n} a_{k} k(k-1) \cdots(k-m+1) t^{k-m}\right. \\
&\left.+\sum_{k=m}^{n} b_{k} k(k-1) \cdots(k-m+1) t^{k-m}\right] \\
& v_{m}= \frac{(x-l)^{2 m}}{\mu_{2}^{m}(2 m) !} \quad(1 \leq m \leq n), \\
& \times\left[\begin{array}{c}
x-l \\
2 m+1
\end{array} \sum_{k=m}^{n} c_{k} k(k-1) \cdots(k-m+1) t^{k-m}\right. \\
&\left.+\sum_{k=m}^{n} d_{k} k(k-1) \cdots(k-m+1) t^{k-m}\right] \\
& v_{m}=0 \quad(m>n) .
\end{aligned}
$$


For subsequent numerical computation, let the expressions

$$
\begin{gathered}
\bar{u}=\bar{u}\left(x, t ; b_{1}, b_{2}, \ldots, b_{n}\right)=\sum_{m=0}^{n} u_{m} \\
\bar{v}=\bar{v}\left(x, t ; c_{1}, c_{2}, \ldots, c_{n} ; d_{1}, d_{2}, \ldots, d_{n}\right)=\sum_{m=0}^{n} v_{m}
\end{gathered}
$$

denote the approximation to $u$ and $v$, respectively. Substituting $\bar{u}$ and $\bar{v}$ in (31) for $u$ and $v$ in (6) and (8) yields

$$
\begin{aligned}
& P\left(t ; c_{1}, c_{2}, \ldots, c_{n} ; d_{1}, d_{2}, \ldots, d_{n}\right) \\
& =\lambda_{2} \frac{\partial \bar{v}}{\partial x}\left(l, t ; c_{1}, c_{2}, \ldots, c_{n} ; d_{1}, d_{2}, \ldots, d_{n}\right) \\
& +\alpha(t)\left[\bar{v}\left(l, t ; c_{1}, c_{2}, \ldots, c_{n} ; d_{1}, d_{2}, \ldots, d_{n}\right)-v^{*}\right]=0 \\
& \left(0 \leq t \leq t^{*}\right) \text {, } \\
& \bar{u}\left(s_{0}, 0 ; b_{1}, b_{2}, \ldots, b_{n}\right)-u^{*}=0, \\
& \bar{v}\left(s_{0}, 0 ; c_{1}, c_{2}, \ldots, c_{n} ; d_{1}, d_{2}, \ldots, d_{n}\right)-u^{*}=0, \\
& \bar{u}\left(s(t), t ; b_{1}, b_{2}, \ldots, b_{n}\right)-u^{*}=0 \quad\left(0<t \leq t^{*}\right), \\
& \bar{v}\left(s(t), t ; c_{1}, c_{2}, \ldots, c_{n} ; d_{1}, d_{2}, \ldots, d_{n}\right)-u^{*}=0 \\
& \left(0<t \leq t^{*}\right) \text {, } \\
& Q\left(s(t), t ; b_{1}, b_{2}, \ldots, b_{n} ; c_{1}, c_{2}, \ldots, c_{n} ; d_{1}, d_{2}, \ldots, d_{n}\right) \\
& =\lambda_{2} \frac{\partial \bar{u}}{\partial x}\left(s(t), t ; b_{1}, b_{2}, \ldots, b_{n}\right) \\
& \times \frac{\partial \bar{v}}{\partial x}\left(s(t), t ; c_{1}, c_{2}, \ldots, c_{n} ; d_{1}, d_{2}, \ldots, d_{n}\right) \\
& -\lambda_{1}\left(\frac{\partial \bar{u}}{\partial x}\right)^{2}\left(s(t), t ; b_{1}, b_{2}, \ldots, b_{n}\right) \\
& +\kappa \frac{\partial \bar{u}}{\partial t}\left(s(t), t ; b_{1}, b_{2}, \ldots, b_{n}\right)=0 \\
& \left(0<t<t^{*}\right) .
\end{aligned}
$$

There are many methods for determining the unknown numbers $b_{1}, b_{2}, \ldots, b_{n}, c_{1}, c_{2}, \ldots, c_{n}$, and $d_{1}, d_{2}, \ldots, d_{n}$ to satisfy (32)-(37). For instance, we can choose different $t_{i} \in\left(0, t^{*}\right)$ $(i=1,2, \ldots, n)$ and substitute these into (32), (35), (36), and (37) to generate the equations

$$
\begin{gathered}
P\left(t_{i} ; c_{1}, c_{2}, \ldots, c_{n} ; d_{1}, d_{2}, \ldots, d_{n}\right)=0 \quad(i=1,2, \ldots, n), \\
\bar{u}\left(s_{i}, t_{i} ; b_{1}, b_{2}, \ldots, b_{n}\right)-u^{*}=0 \quad(i=1,2, \ldots, n), \\
\bar{v}\left(s_{i}, t_{i} ; c_{1}, c_{2}, \ldots, c_{n} ; d_{1}, d_{2}, \ldots, d_{n}\right)-u^{*}=0 \\
(i=1,2, \ldots, n),
\end{gathered}
$$

$$
\begin{array}{r}
Q\left(s_{i}, t_{i} ; b_{1}, b_{2}, \ldots, b_{n} ; c_{1}, c_{2}, \ldots, c_{n} ; d_{1}, d_{2}, \ldots, d_{n}\right)=0 \\
(i=1,2, \ldots, n),
\end{array}
$$

where $s_{i}=s\left(t_{i}\right)$. Then (33), (34), and (38) constitute a system of nonlinear equations in $4 n$ unknowns, $b_{1}, b_{2}, \ldots, b_{n}$, $c_{1}, c_{2}, \ldots, c_{n}, d_{1}, d_{2}, \ldots, d_{n}$, and $s_{1}, s_{2}, \ldots, s_{n}$, and $4 n+2$ equations. Solving this system, we can obtain the leastsquares solutions of the system. Then substituting the known numbers $b_{1}, b_{2}, \ldots, b_{n}, c_{1}, c_{2}, \ldots, c_{n}$, and $d_{1}, d_{2}, \ldots, d_{n}$ into (31), we can obtain the approximate analytic solutions $\bar{u}=$ $\bar{u}(x, t)$ and $\bar{v}=\bar{v}(x, t)$ and the equation $\bar{u}(s, t)-u^{*}=0$, which determines the moving boundary $s=s(t)$ in the form of an implicit function.

\section{Computation Using MATLAB}

To solve the two-phase Stefan problem (1)-(7), we decompose the operation into a series of suboperations including expansion of functions into the Taylor series, differentiation, integration, substitution, and solution of a system of nonlinear equations. These suboperations are easily implemented using computing software; we chose MATLAB as the tool for mathematical operations.

To show how to implement the operations in Section 3, a concrete two-phase Stefan problem [18] is solved in which the parameters $\mu_{1}=2.5, \mu_{2}=1.25, s_{0}=1.5, l=3, t^{*}=1.5$, $\lambda_{1}=6, \lambda_{2}=2, \kappa=0.8, u^{*}=1$, and $v^{*}=0.9$ are assumed. The functions for the initial and boundary conditions are as follows:

$$
\begin{gathered}
\varphi(x)=e^{-0.2 x+0.3}, \\
\psi(x)=e^{-0.4 x+0.6}, \\
q(t)=1.2 e^{0.1 t+0.3}, \\
\alpha(t)=\frac{0.8 e^{0.2 t-0.6}}{e^{0.2 t-0.6}-0.9} .
\end{gathered}
$$

Accordingly, the exact solutions of the model (1)-(7) are $u(x, t)=e^{-0.2 x+0.1 t+0.3}, v(x, t)=e^{-0.4 x+0.2 t+0.6}$, and $s(t)=$ $0.5 t+1.5$.

Using (19), (20), (23), (39), (40), and (42), we obtain $b_{0}=e^{0.3}, c_{0}=-0.21952465$, and $d_{0}=e^{-0.6}$. The choice of polynomial degree $n$ in (17) and (18) is important for solving the model. If $n$ is too small, the precision of $\bar{u}=\bar{u}(x, t)$ and $\bar{v}=\bar{v}(x, t)$ will not be high; if $n$ is too large, solving the nonlinear system of equations constituted by (33), (34), and (38) will be difficult. Considering these two factors, we choose $n=6$. According to (12), (14), (17), and (41), we choose the sixth-order Taylor approximation to $-0.2 e^{0.1 t+0.3}$ as $\sum_{k=0}^{6} a_{k} t^{k}$. Computing the expansion using the MATLAB function taylor( ) yields

$$
\begin{aligned}
& \sum_{k=0}^{6} a_{k} t^{k} \\
& \quad=-e^{0.3}\left(\frac{1}{5}+\frac{1}{50} t+\frac{1}{1000} t^{2}+\cdots+\frac{1}{3600000000} t^{6}\right) .
\end{aligned}
$$


The recursive operation in (28) and (29) contains differential and integral polynomials that can easily be obtained using the MATLAB functions $\operatorname{diff}()$ and $\operatorname{int}()$. Thus, $\bar{u}=$ $\bar{u}\left(x, t ; b_{1}, b_{2}, \ldots, b_{6}\right)=\sum_{m=0}^{6} u_{m}$ and $\bar{v}=\bar{v}\left(x, t ; c_{1}, c_{2}, \ldots\right.$, $\left.c_{6} ; d_{1}, d_{2}, \ldots, d_{6}\right)=\sum_{m=0}^{6} v_{m}$ in (31) were determined. Taking $t_{1}=0.2, t_{2}=0.4, t_{3}=0.6, t_{4}=0.8, t_{5}=1.0$, and $t_{6}=1.2 \mathrm{in}$ the interval $(0,1.5)$ and using the MATLAB functions $\operatorname{diff(}()$ and subs( ), we can obtain the following algebraic system of equations:

$$
\begin{aligned}
& \bar{u}\left(1.5,0 ; b_{1}, b_{2}, \ldots, b_{6}\right)-1=0, \\
& \bar{v}\left(1.5,0 ; c_{1}, c_{2}, \ldots, c_{6} ; d_{1}, d_{2}, \ldots, d_{6}\right)-1=0, \\
& P\left(0.2 i ; c_{1}, c_{2}, \ldots, c_{6} ; d_{1}, d_{2}, \ldots, d_{6}\right)=0 \quad(i=1,2, \ldots, 6), \\
& \bar{u}\left(s_{i}, 0.2 i ; b_{1}, b_{2}, \ldots, b_{6}\right)-1=0 \quad(i=1,2, \ldots, 6), \\
& \bar{v}\left(s_{i}, 0.2 i ; c_{1}, c_{2}, \ldots, c_{6} ; d_{1}, d_{2}, \ldots, d_{6}\right)-1=0 \\
& \quad(i=1,2, \ldots, 6), \\
& Q\left(s_{i}, 0.2 i ; b_{1}, b_{2}, \ldots, b_{6} ; c_{1}, c_{2}, \ldots, c_{6} ; d_{1}, d_{2}, \ldots, d_{6}\right)=0 \\
& \quad(i=1,2, \ldots, 6),
\end{aligned}
$$

which is determined by (33), (34), and (38).

Solving (44) yields

$$
\begin{aligned}
& \left(b_{1}, b_{2}, b_{3}, b_{4}, b_{5}, b_{6} ; c_{1}, c_{2}, c_{3},\right. \\
& \left.c_{4}, c_{5}, c_{6} ; d_{1}, d_{2}, d_{3}, d_{4}, d_{5}, d_{6}\right) \\
& =(0.134986,0.006750,0.002245, \\
& \quad 0.000006,0.000000, \\
& \quad 0.000000 ;-0.043904,-0.004395,-0.000283, \\
& \quad-0.000026,0.000005,-0.000001 ; \\
& \quad 0.109763,0.010972, \\
& \quad 0.000741,0.000027,0.0000063,-0.000001), \\
& \left(s_{1}, s_{2}, s_{3}, s_{4}, s_{5}, s_{6}\right)=(1.6,1.7,1.8,1.9,2.0,2.1) .
\end{aligned}
$$

Then the expressions for $\bar{u}=\bar{u}(x, t)$ and $\bar{v}=\bar{v}(x, t)$ are known. These expressions are very long so we do not explicitly present them here and instead we show only plots of the absolute error functions $|u(x, t)-\bar{u}(x, t)|$ and $\mid v(x, t)-$ $\bar{v}(x, t) \mid$.

As shown in Figures 2 and 3, the accuracy of the approximate analytic solutions $u=\bar{u}(x, t)$ and $v=\bar{v}(x, t)$ is of the order of at least $10^{-5}$. This will result in the same high accuracy for the approximate solution for the moving boundary $x=\bar{s}(t)$ as that determined by the implicit function $\bar{u}(x, t)-u^{*}=0$.

\section{Conclusion}

When the solutions and boundary condition functions for PDEs and systems thereof are smooth enough, they can be

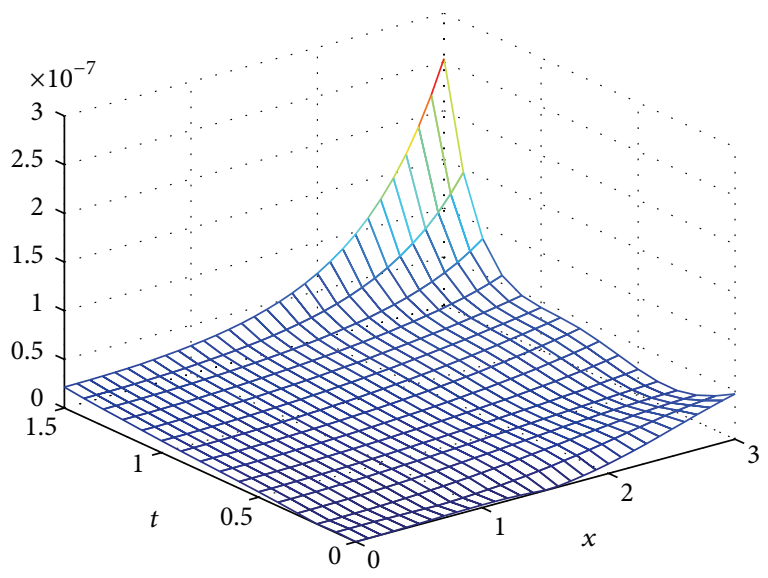

Figure 2: Plot of the absolute error functions $|u(x, t)-\bar{u}(x, t)|$ for the exact solution $u(x, t)=e^{-0.2 x+0.1 t+0.3}$.

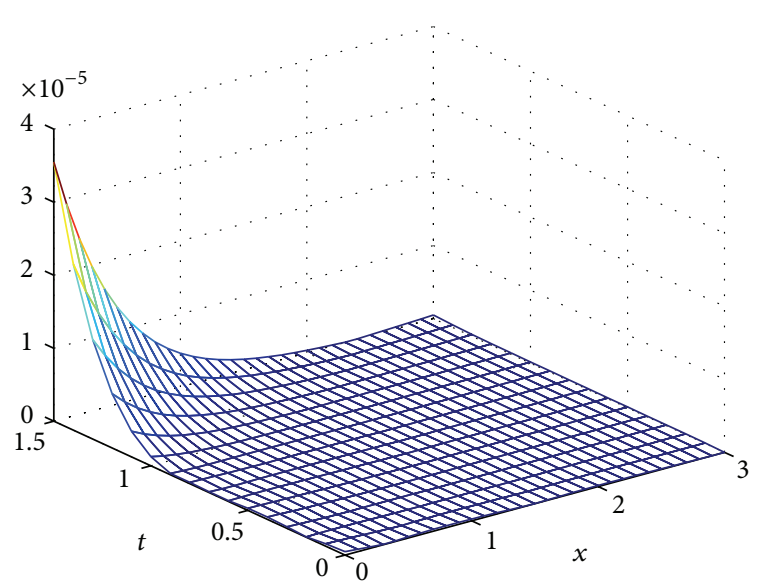

FIGURE 3: Plot of the absolute error functions $|v(x, t)-\bar{v}(x, t)|$ for the exact solution $v(x, t)=e^{-0.4 x+0.2 t+0.6}$.

approximated by polynomials. Therefore, only differential, integral, and substitution operations for polynomials and other simple elementary functions are required to identify expressions for the approximate analytic solutions of equations or a system of equations using ADM. To find solutions satisfying all the given equations and conditions for a Stefan problem, we need to solve a nonlinear system of equations. This is like solving a PDE using finite element and finite difference methods. However, compared to these traditional methods, the proposed approach has faster convergence and higher-order accuracy and can give approximate expressions for solutions. This is an efficient method for finding approxmate analytic solutions for the Stefan problems using scientific software.

\section{Conflict of Interests}

The authors declare that there is no conflict of interests regarding the publication of this paper. 


\section{References}

[1] J. Crank, Free and Moving Boundary Problems, Clarendon Press, Oxford, UK, 1984.

[2] A. M. Meirmanov, The Stefan Problem, Walter de Gruyter, Berlin, Germany, 1992.

[3] O. Levenspiel, Chemical Reaction Engineering, John Wiley \& Sons, New York, NY, USA, 3rd edition, 1999.

[4] V. Srinivasan and J. Newman, "Discharge model for the lithium iron-phosphate electrode," Journal of the Electrochemical Society, vol. 151, no. 10, pp. A1517-A1529, 2004.

[5] L. N. Tao, "A method for solving moving boundary problems," SIAM Journal on Applied Mathematics, vol. 46, no. 2, pp. 254264, 1986.

[6] G. F. Carey and P. Murray, "Perturbation analysis of the shrinking core," Chemical Engineering Science, vol. 44, no. 4, pp. 979-983, 1989.

[7] S. Chen, B. Merriman, S. Osher, and P. Smereka, "A simple level set method for solving Stefan problems," Journal of Computational Physics, vol. 135, no. 1, pp. 8-29, 1997.

[8] D. Słota, "Direct and inverse one-phase Stefan problem solved by the variational iteration method," Computers \& Mathematics with Applications, vol. 54, no. 7-8, pp. 1139-1146, 2007.

[9] F. Gibou and R. Fedkiw, "A fourth order accurate discretization for the Laplace and heat equations on arbitrary domains, with applications to the Stefan problem," Journal of Computational Physics, vol. 202, no. 2, pp. 577-601, 2005.

[10] R. H. Nochetto, M. Paolini, and C. Verdi, "An adaptive finite element method for two-phase Stefan problems in two space dimensions. I: stability and error estimates," Mathematics of Computation, vol. 57, no. 195, pp. 73-108, 1991.

[11] R. Robalo, C. A. Sereno, M. C. Coimbra, and A. E. Rodrigues, "The numerical solution for moving boundary problem using the moving finite element method," in Proceedings of the European Symposium on Computer Aided Process Engineering, Elsevier Science, 2005.

[12] G. Adomian, Solving Frontier Problems of Physics: The Decomposition Method, vol. 60 of Fundamental Theories of Physics, Kluwer Academic Publishers, Dodrecht, The Netherlands, 1994.

[13] G. Adomian, "Solution of coupled nonlinear partial differential equations by decomposition," Computers \& Mathematics with Applications, vol. 31, no. 6, pp. 117-120, 1996.

[14] L. Bougoffa, "Adomian method for a class of hyperbolic equations with an integral condition," Applied Mathematics and Computation, vol. 177, no. 2, pp. 545-552, 2006.

[15] J.-S. Duan and R. Rach, "A new modification of the Adomian decomposition method for solving boundary value problems for higher order nonlinear differential equations," Applied Mathematics and Computation, vol. 218, no. 8, pp. 4090-4118, 2011.

[16] J.-S. Duan and R. Rach, "New higher-order numerical one-step methods based on the Adomian and the modified decomposition methods," Applied Mathematics and Computation, vol. 218, no. 6, pp. 2810-2828, 2011.

[17] S. M. El-Sayed, "The modified decomposition method for solving nonlinear algebraic equations," Applied Mathematics and Computation, vol. 132, no. 2-3, pp. 589-597, 2002.

[18] R. Grzymkowski, M. Pleszczyński, and D. Słota, "The two-phase Stefan problem solved by the Adomian decomposition method," in Proceedings of the 15th IASTED International Conference on Applied Simulation and Modelling, pp. 511-516, Rhodes, Greece, June 2006.
[19] R. Grzymkowski and D. Słota, "Stefan problem solved by Adomian decomposition method," International Journal of Computer Mathematics, vol. 82, no. 7, pp. 851-856, 2005.

[20] X. Y. Qin and Y. P. Sun, "Approximate analytic solutions for a two-dimensional mathematical model of a packed-bed electrode using the Adomian decomposition method," Applied Mathematics and Computation, vol. 215, no. 1, pp. 270-275, 2009.

[21] X. Y. Qin and Y. P. Sun, "Approximate analytical solutions for a mathematical model of a tubular packed-bed catalytic reactor using an Adomian decomposition method," Applied Mathematics and Computation, vol. 218, no. 5, pp. 1990-1996, 2011.

[22] X. Y. Qin and Y. P. Sun, "Approximate analytical solutions for a shrinking core model for the discharge of a lithium ironphosphate electrode by the Adomian decomposition method," Applied Mathematics and Computation, vol. 230, pp. 267-275, 2014.

[23] A.-M. Wazwaz, Partial Differential Equations and Solitary Waves Theory, Nonlinear Physical Science, Springer, Berlin, Germany, 2009.

[24] S. Das and Rajeev, "An approximate analytical solution of onedimensional phase change problems in a finite domain," Applied Mathematics and Computation, vol. 217, no. 13, pp. 6040-6046, 2011. 


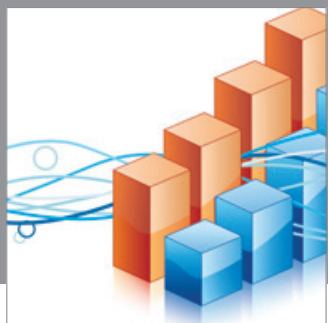

Advances in

Operations Research

mansans

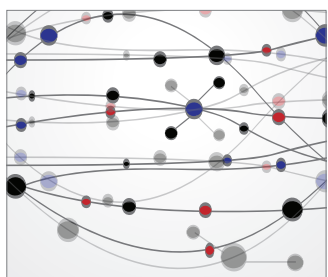

The Scientific World Journal
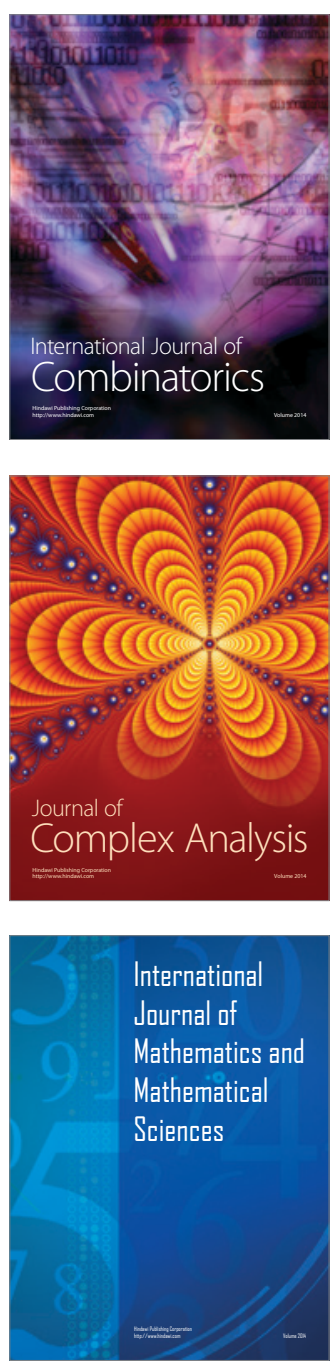
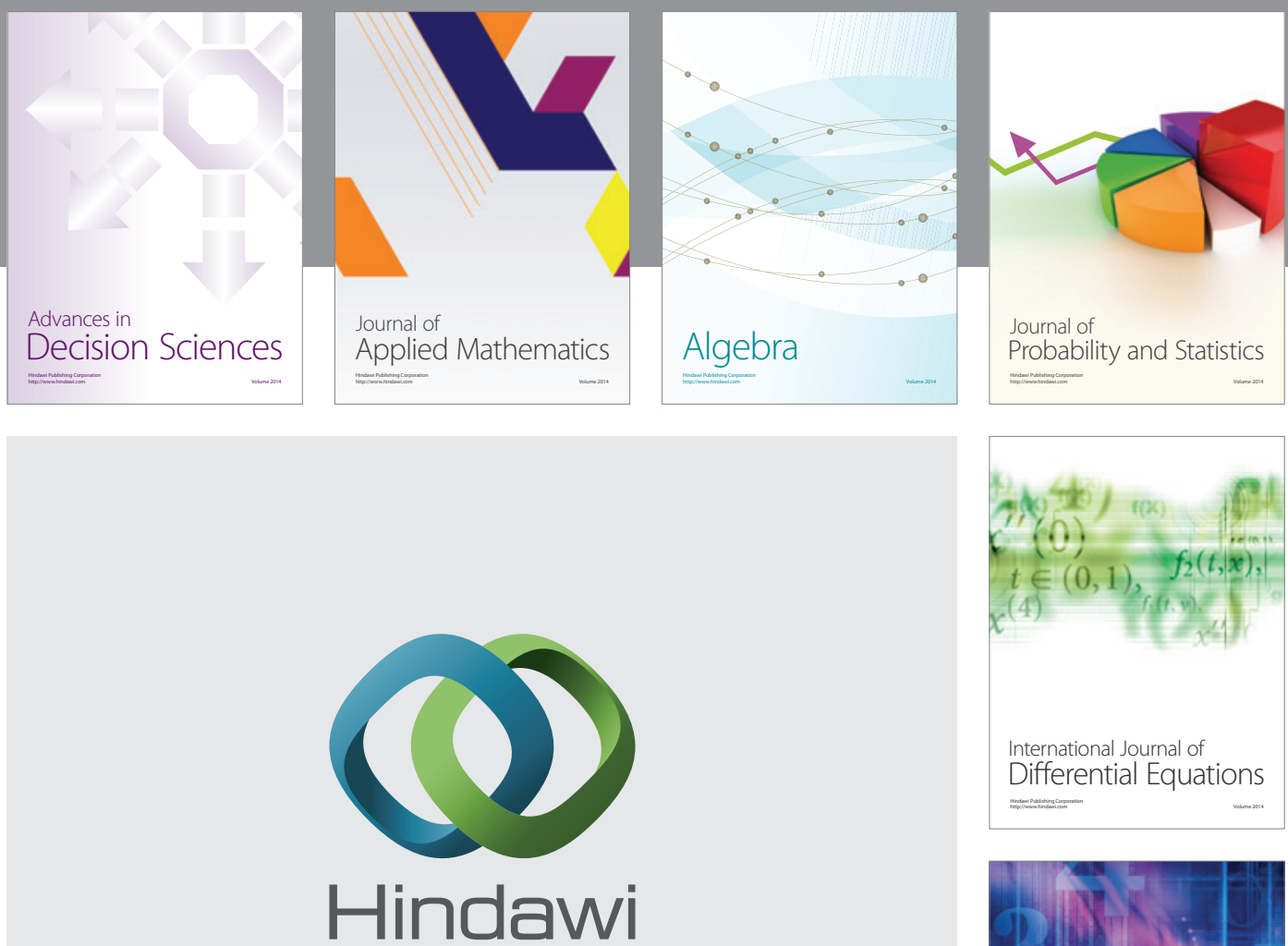

Submit your manuscripts at http://www.hindawi.com
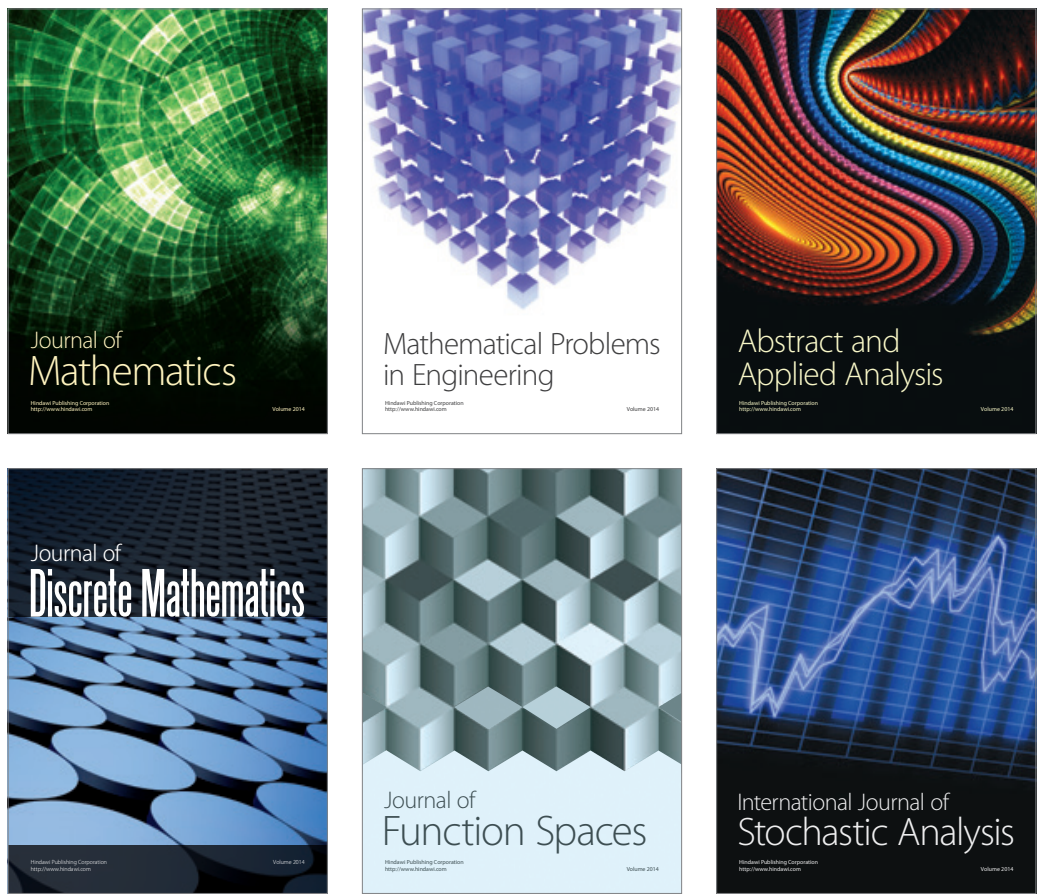

Journal of

Function Spaces

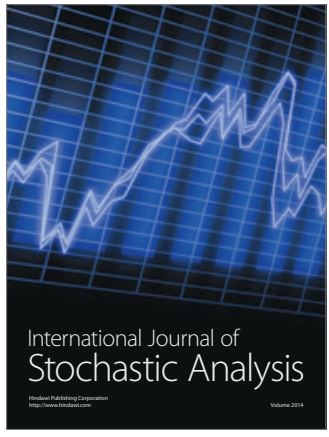

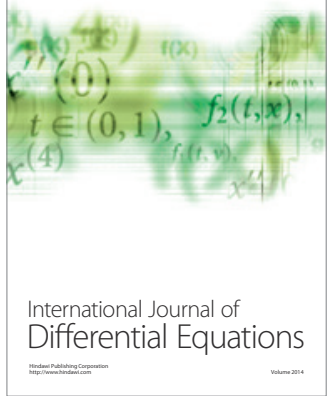
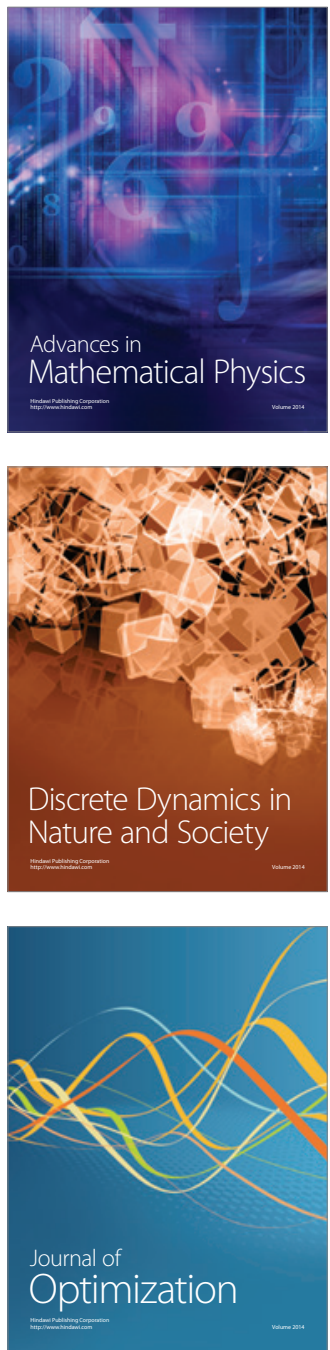